\title{
EDITORIAL
}

\section{Quince años de la revista Maderas-Ciencia y tecnología: De la infancia a la adolescencia}

\author{
Rubén A. ANANIAS \\ Director-Editor
}

Quince años para una revista científica es una edad que estimo puede ser considerada como equivalente a la evolución de la infancia a la adolescencia. Las capacidades aprehendidas durante la infancia ya quedaron instaladas para desarrollarse en esta nueva etapa.

Este final del ciclo infantil encuentra a la revista Maderas-Cienc Tecnol en una posición histórica, frente a la revelación del Journal Citation Report ${ }^{\circledR}$, Science Edition 2012, alcanzando un Thomson Reuters Impact Factor de 0.722 y siendo rankeada entre las primeras 10 revistas y en el cuartil Q2 de la categoría Materials Science, Paper $\&$ Wood (Tabla 1).

Tabla 1. Factor de impacto, ranking y cuartil de algunas revistas de la categoría Materials, Science: Paper\&Wood (Fuente: 2012 JCR Science Edition)

\begin{tabular}{|l|c|c|c|}
\hline Nombre de la revista & Factor de impacto & Ranking & Cuartil \\
\hline Wood Sci Technol & 1.884 & 3 & Q1 \\
\hline Maderas-Cienc Tecnol & 0.722 & 10 & Q2 \\
\hline Forest Prod J & 0,347 & 15 & Q3 \\
\hline Wood Research & 0,275 & 17 & Q4 \\
\hline
\end{tabular}

Como Editor, concuerdo con que el factor de impacto de Thomson Reuters debidamente utilizado es un buen estimador de la calidad e influencia de una revista científica (Nature Materials 2013). En este sentido la revista Maderas-Cienc Tecnol presenta un 10\% de autocitas, lejos del 19\% que alcanza la mediana de la categoría Materials Science, Paper \& Wood (2012 JCR Science Edition).

Volviendo a la evolución de la revista, atrás quedaron la gestación y el nacimiento propiamente tal en el curso del año 1998. Los pioneros, tuvieron la visión que la revista Maderas-Cienc Tecnol contribuiría al desarrollo de una sociedad basada en el conocimiento (Hernández 1998).

A continuación, la ablactación limitando la endogamia, dando los primeros pasos y llamando la atención del mundo, luego la necesidad de diferenciarse de sus pares de los 3 años, la capacidad para ir incorporando reglas y normas, tal como las primeras palabras claves a los 5 años, al ingresar en el Forest Product Abstract en el año 2003. El aprendizaje y obedecimiento de las reglas de los 7 años, incorporando nuevas metodologías para la publicación online en la biblioteca científica Scielo en el curso del año 2005. La consolidación de una identidad científica propia como revista y el reconocimiento externo de las potencialidades a los 10 años, las innumerables felicitaciones, como nunca anteriormente, por el acceso de la revista Maderas-Cienc Tecnol a ISI-WoS de Thomson Reuters durante el año 2008 (Ananías 2008). 
A los 13 años se asumía una mayor responsabilidad científica y se esperaba con expectación conocer el primer factor de impacto del Journal Citation Report@ del año 2010, se había estimado en 0.194 y se alcanzó 0.327, en promedio uno de cada tres artículos de la revista Maderas-Cienc Tecnol estaban siendo citados por otras revistas científicas internacionales. Fortalecíamos durante ese mismo período la visibilidad internacional de la revista (Ananías y Salvo 2010).

En perspectiva, durante estos quince años la revista Maderas-Cienc Tecnol, ha estado evolucionando de la infancia a la adolescencia, con entusiasmo y dinamismo: Piedra movediza moho no cobija. De cara al futuro, se espera continuar progresando como revista de investigación científica, seguir contando con la confianza de los autores que desean diseminar sus hallazgos científicos y tecnológicos en el ámbito de las Ciencias de la Madera. En fin, los quince años cumplidos, servirán de base para alcanzar el apogeo de la juventud: comunicar ciencia de alto impacto con visibilidad internacional y excelencia científica.

\section{REFERENCIAS}

Ananías, R.A. 2008. Diez años después: La fluidez de la revista MCT. Maderas. Ciencia y tecnología 10(3):183184.

Ananías, R.A.; Salvo, L. 2010. Fortalecimiento de la visibilidad de la revista Maderas-Ciencia y tecnología. Poster presentado en II Congreso Internacional de Editores Redalyc, 16-19 noviembre, 2010, Valdivia, Chile.

Hernández, H. 1998. El mañana es la edad de la madera. Maderas. Ciencia y tecnología 1(1):3-4.

Nature Materials. 2013. Beware the impact factor. Nature Materials 12(2):89. 